\title{
Evolution of axions in the presence of primordial magnetic fields
}

\author{
Maxim Dvornikov@* and V. B. Semikoz $\oplus^{\dagger}$ \\ Pushkov Institute of Terrestrial Magnetism, Ionosphere and Radiowave Propagation (IZMIRAN), \\ 108840 Moscow, Troitsk, Russia
}

(Received 2 October 2020; accepted 20 November 2020; published 15 December 2020)

\begin{abstract}
We study the evolution of axions interacting with primordial magnetic fields (PMFs) starting from the QCD phase transition in the expanding Universe. This interaction is owing to the Primakoff effect. Adopting the zero-mode approximation for axions, we derive the system of equations for axions and magnetic fields, where the expansion of the Universe and the spectra of magnetic fields are accounted for exactly. We find that the contribution of the Primakoff effect to the dynamics of axions and magnetic fields is rather weak. This confirms some previous estimates leading to analogous conclusions when we account for the Hubble expansion for both a uniform axion field and nonuniform PMFs using Fourier spectra for their energy and helicity densities. We solve the corresponding system of the evolution equations and find that the amplitude of the axion zero mode, when evolving during the radiation era, is at a level sufficient for the axion to be a good candidate for cold dark matter.
\end{abstract}

DOI: 10.1103/PhysRevD.102.123526

\section{INTRODUCTION}

As a solution to the $C P$ problem in quantum chromodynamics (QCD), Peccei and Quinn suggested a mechanism that naturally gives rise to a Nambu-Goldstone boson, the so-called axion [1-3]. In addition, the axion also provides a possible candidate for the cold dark matter (CDM) of the Universe [4]. There are a number of active searches for axions [5], and progress in the experimental studies of these particles was recently reported in Ref. [6].

The simultaneous presence of primordial magnetic fields (PMFs) and an axion field in a hot Universe plasma near the QCD phase transition (QCDPT) allows to study their mutual influence during the radiation era. We adopt that in the Universe cooling $T_{\mathrm{PQ}} \rightarrow T_{\mathrm{QCD}}$, where $T_{\mathrm{PQ}} \simeq f_{a}=$ $\left(10^{10}-10^{12}\right) \mathrm{GeV}$ is the temperature at the Peccei-Quinn phase transition, $T_{\mathrm{QCD}} \simeq(100-200) \mathrm{MeV}$ is the temperature at the QCDPT, and $f_{a}$ is the Peccei-Quinn (PQ) parameter. The small axion mass $m_{a}(T)$ increases and gets its "cold" (fixed) value $m_{a}(0)=m_{a}$ after mixing with $\pi^{0}$ mesons near the initial time in our problem, $t_{0}=t_{\mathrm{QCD}}$,

$$
m_{a}=6 \times 10^{-6} \mathrm{eV}\left(\frac{10^{12} \mathrm{GeV}}{f_{a}}\right) .
$$

*maxdvo@izmiran.ru

†semikoz@yandex.ru

Published by the American Physical Society under the terms of the Creative Commons Attribution 4.0 International license. Further distribution of this work must maintain attribution to the author(s) and the published article's title, journal citation, and DOI. Funded by SCOAP ${ }^{3}$.
In its turn, PMFs originate from the hypermagnetic fields $B_{\mathrm{Y}}$ which exist before the electroweak phase transition, $T>T_{\mathrm{EW}} \simeq$ (100-160) GeV. Such PMFs, being a seed for observable galactic magnetic fields $B \sim 10^{-6} \mathrm{G}$, can be strong enough at $T_{\mathrm{QCD}}$. If we adopt the present bound on the extragalactic PMF, $B \leq B_{\text {now }}=10^{-9} \mathrm{G}$, it corresponds to the PMF strength $B_{\mathrm{QCD}}=B_{\text {now }}\left(1+z_{\mathrm{QCD}}\right)^{2} \simeq 1.6 \times$ $10^{14} \mathrm{G}$ in the early Universe. Here we use the red shift $z_{\mathrm{QCD}}=4 \times 10^{11}$ at $T_{\mathrm{QCD}} \sim 100 \mathrm{MeV}$, or $B_{\mathrm{EW}}=$ $1.6 \times 10^{20} \mathrm{G}$ at $T_{\mathrm{EW}} \sim 100 \mathrm{GeV}$.

On the one hand, such a strong field cannot influence the Universe's expansion since the PMF energy density $\rho_{\mathrm{B}}=$ $B^{2} / 2$ is much less than the ultrarelativistic matter energy density $\rho=g^{*} \pi^{2} T^{4} / 30$, that is, $\rho_{\mathrm{B}} \ll \rho$. Here $g^{*}$ is the effective number of degrees of freedom [[7], p. 95]. On the other hand, it is intriguing to check whether such a field can be enlarged by the axion instability term $\sim \dot{\varphi} \nabla \times \mathbf{B}$ entering the induction equation [8],

$$
\frac{\partial \mathbf{B}}{\partial t}=\frac{\nabla^{2} \mathbf{B}}{\sigma_{\text {cond }}}+\frac{g_{a \gamma}}{\sigma_{\text {cond }}} \frac{\mathrm{d} \varphi}{\mathrm{d} t} \nabla \times \mathbf{B},
$$

or, vice versa, whether the PMF can influence the evolution of the uniform axion field, $\nabla \varphi=0$,

$$
\ddot{\varphi}+m_{a}^{2} \varphi=\frac{g_{a \gamma}}{\sigma_{\text {cond }}}(\nabla \times \mathbf{B}) \cdot \mathbf{B} .
$$

Here $g_{a \gamma} \approx \alpha_{\mathrm{em}} / 2 \pi f_{a}$ is the coupling constant for axion interactions with electromagnetic fields, $\alpha_{\mathrm{em}} \approx(137)^{-1}$ is the fine-structure constant, and $\sigma_{\text {cond }} \simeq 100 T$ is the electric conductivity in the hot Universe plasma. 
The problem of the mutual influence of axions and PMFs was discussed in Ref. [8]. However, in Ref. [8] the Hubble expansion was neglected for both a uniform axion gas and primordial magnetic fields. We study a similar problem by accounting for both the expansion when using conformal variables and an inhomogeneity of PMFs through their Kolmogorov spectra for the densities of the magnetic energy and magnetic helicity.

Our work is organized as follows. In Sec. II we present the complete system of evolution equations for the homogeneous axion field $\varphi(\eta)$, and the spectra of the conformal PMF energy density $\rho_{c}\left(k_{c}, \eta\right)$ and the conformal PMF helicity density $h_{c}\left(k_{c}, \eta\right)$. Here $\eta$ is the conformal time and $k_{c}$ is the conformal momentum. Details for the derivation of such a system are given in the Appendix. In Sec. II B we formulate the initial conditions for this system of evolution equations. In Sec. III we present the results of the numerical calculations, illustrated by plots both for the maximum extragalactic field $B_{\text {now }}=10^{-9} \mathrm{G}$ and without PMFs at all. In Sec. IV we discuss our results.

\section{AXIONS IN THE EARLY UNIVERSE PLASMA WITH PMFs}

For a homogeneous axion field $\nabla \varphi=0$, or for the zero axion mode [9], the axion evolution equation in the presence of PMFs and in the Friedmann-Robertson-Walker (FRW) metric, $\mathrm{d} s^{2}=a^{2}(t)\left(\mathrm{d} \eta^{2}-\mathrm{d} \mathbf{x}^{2}\right)$, where $a$ is the scale factor and $\mathrm{d} \eta=d t / a(t)$, takes the following form in the variables $x^{\mu}=(\eta, \mathbf{x})$ [see Eqs. (A7) and (A8) in the Appendix]:

$$
\frac{\mathrm{d}^{2} \varphi}{\mathrm{d} \eta^{2}}+\frac{2}{a} \frac{\mathrm{d} a}{\mathrm{~d} \eta} \frac{\mathrm{d} \varphi}{\mathrm{d} \eta}+m_{a}^{2} a^{2} \varphi=-\frac{g_{a \gamma}}{2 a^{2}} \frac{\mathrm{d} h_{c}(\eta)}{\mathrm{d} \eta} .
$$

Here $h_{c}(\eta)=a^{3} h(t)=V^{-1} \int \mathrm{d}^{3} x \mathbf{A}_{c}(\eta, \mathbf{x}) \cdot \mathbf{B}_{c}(\eta, \mathbf{x})$ is the conformal PMF helicity density, $\mathbf{A}_{c}(\eta, \mathbf{x})=a \mathbf{A}$ is the vector potential, and $\mathbf{B}_{c}(\eta, \mathbf{x})=\nabla \times \mathbf{A}_{c}(\eta, \mathbf{x})=a^{2} \mathbf{B}$ is the comoving magnetic field in conformal variables [11].

We can calculate the derivative $\mathrm{d} h_{c}(\eta) / \mathrm{d} \eta$ for the magnetic helicity density $h_{c}(\eta)=\int \mathrm{d} k_{c} h\left(\eta, k_{c}\right)$ given by its isotropic Fourier spectrum $h_{c}(k, \eta)=k_{c}^{2} \mathbf{A}_{c}\left(k_{c}, \eta\right) \mathbf{B}_{c}^{*}\left(k_{c}, \eta\right) /$ $\left(2 \pi^{2} V\right)$ as $\partial_{\eta} h_{c}(\eta)=\int \mathrm{d} k_{c} \partial_{\eta} h_{c}\left(\eta, k_{c}\right)$, where $k_{c}=a k=$ const is the comoving momentum that does not depend on time. We use the evolution equations for PMF spectra for this purpose (see the Appendix),

$$
\begin{aligned}
& \frac{\partial h_{c}\left(k_{c}, \eta\right)}{\partial \eta}=-\frac{2 k_{c}^{2}}{\sigma_{c}} h_{c}\left(k_{c}, \eta\right)+\frac{4 g_{a \gamma}}{\sigma_{c}} \frac{\mathrm{d} \varphi(\eta)}{\mathrm{d} \eta} \rho_{c}\left(k_{c}, \eta\right), \\
& \frac{\partial \rho_{c}\left(k_{c}, \eta\right)}{\partial \eta}=-\frac{2 k_{c}^{2}}{\sigma_{c}} \rho_{c}\left(k_{c}, \eta\right)+k_{c}^{2} \frac{g_{a \gamma}}{\sigma_{c}} \frac{\mathrm{d} \varphi(\eta)}{\mathrm{d} \eta} h_{c}\left(k_{c}, \eta\right),
\end{aligned}
$$

where the magnetic energy density $\rho_{c}(\eta)=\int \mathrm{d} k_{c} \rho_{c}\left(\eta, k_{c}\right)=$ $B_{c}^{2}(\eta) / 2$ is given by the isotropic Fourier spectrum $\rho_{c}\left(k_{c}, \eta\right)=k_{c}^{2} \mathbf{B}_{c}\left(k_{c}, \eta\right) \mathbf{B}_{c}^{*}\left(k_{c}, \eta\right) /\left(4 \pi^{2} V\right), k_{c}=\left|\mathbf{k}_{c}\right|$ is the absolute value of the comoving Fourier momentum, and $\sigma_{c}=a \sigma_{\text {cond }}$ is given by the electric conductivity $\sigma_{\text {cond }} \simeq$ $100 T$ in the hot Universe plasma.

Substituting the derivative $\mathrm{d} h_{c}(\eta) / \mathrm{d} \eta=$ $\int_{k_{\min }}^{k_{\max }} \mathrm{d} k_{c} \partial_{\eta} h_{c}\left(\eta, k_{c}\right)$, with the integrand given by Eq. (2.2), we can rewrite the axion evolution equation (2.1) as

$$
\begin{aligned}
& \frac{\mathrm{d}^{2} \varphi(\eta)}{\mathrm{d} \eta^{2}}+\frac{2}{a(\eta)} \frac{\mathrm{d} \varphi(\eta)}{\mathrm{d} \eta}\left(\frac{\mathrm{d} a(\eta)}{\mathrm{d} \eta}+\frac{g_{a \gamma}^{2}}{a(\eta) \sigma_{c}} \int_{k_{\min }}^{k_{\max }} \rho_{c}\left(k_{c}, \eta\right) \mathrm{d} k_{c}\right) \\
& \quad+m_{a}^{2} a^{2}(\eta) \varphi(\eta) \\
& \quad=\frac{g_{a \gamma}}{a^{2}(\eta) \sigma_{c}} \int_{k_{\min }}^{k_{\max }} k_{c}^{2} h_{c}\left(k_{c}, \eta\right) \mathrm{d} k_{c}
\end{aligned}
$$

which is the generalization of the axion wave equation given in Ref. [8] [their Eq. (40a)] accounting for the Hubble expansion and the Fourier spectra of nonuniform electromagnetic fields.

The system of equations that describes the evolution of the axion field in a PMF, given by Eq. (2.4), is completed by the differential equations (2.2) and (2.3) for the spectra $h_{c}\left(k_{c}, \eta\right)$ and $\rho_{c}\left(k_{c}, \eta\right)$. Here, in the causal scenario, the momentum limits $k_{\min }=k_{1} a(t)$ and $k_{\max }=k_{2} a(t)$ obey the inequality $k_{2}>k_{1}=l_{\mathrm{H}}^{-1}\left(T_{0}\right)=$ const, where the horizon size $l_{\mathrm{H}}\left(T_{0}\right)$ is chosen at the QCD phase transition time $t_{0}=\left(T_{0} / \mathrm{MeV}\right)^{-2} \mathrm{~s}$ when the axion mass $m_{a}(T)$ reaches its maximum ("cold") value, given in Eq. (1.1), during the cooling of the Universe, $T \rightarrow T_{0}$. We consider the time $t_{0} \simeq 10^{-4} \mathrm{~s}$ as the initial moment in our simulations. Correspondingly, for the conformal time during the radiation epoch, $\eta(t)=\int_{t_{0}}^{t} \mathrm{~d} t / a(t)+\eta\left(t_{0}\right)$, for which $a(t)=$ $a\left(t_{0}\right) \sqrt{t / t_{0}}$ is the scale factor in the FRW metric, we put the initial value $\eta\left(t_{0}\right)=0$, resulting in

$$
\eta(t)=\frac{2 t_{0}}{a\left(t_{0}\right)}\left[\sqrt{\frac{t}{t_{0}}}-1\right]
$$

where the initial scale factor $a\left(t_{0}\right)=T_{\text {now }} / T_{0}=\left(1+z_{\mathrm{QCD}}\right)^{-1}$ is given by the redshift at the QCD phase transition, $z_{\mathrm{QCD}} \simeq 4 \times 10^{11}$, since $T_{\text {now }}=2.725 \mathrm{~K}$ and $T_{0}=T_{\mathrm{QCD}}=$ $10^{12} \mathrm{~K} \simeq 100 \mathrm{MeV}$.

We assume that PMF survives against the ohmic diffusion in the early Universe in a wide region of Fourier momenta $k_{1}<k_{2}$. Obviously, such a field survives for the minimal and fixed value $k_{1}=l_{\mathrm{H}}^{-1}=(2 / 3) \times 10^{-20} \mathrm{GeV}$ given by the horizon size $l_{\mathrm{H}}$, or the maximum PMF scale $\Lambda_{\mathrm{B}}^{(\max )}=l_{\mathrm{H}}$ at the initial time $t_{0}$. Additionally, we demand that the expansion time $t=t_{0}\left(T_{0} / T\right)^{2}$ be faster than the diffusion time $t_{\text {diff }}=\sigma_{\text {cond }}\left(\Lambda_{\mathrm{B}}^{(\min )}\right)^{2}$, or $t<t_{\text {diff }}$ even for the minimal PMF scale $\Lambda_{\mathrm{B}}^{(\mathrm{min})}=k_{2}^{-1}$. For the initial time $t_{0}$ this means 


$$
t_{0} \leq \frac{\sigma_{c}}{a\left(t_{0}\right) k_{2}^{2}}, \quad T \leq T_{0},
$$

where the conductivity $\sigma_{\text {cond }}=\sigma_{c} / a\left(t_{0}\right)$ was substituted.

The system of the evolution equations (2.3)-(2.4), rewritten for the three dimensionless functions $\mathcal{H}(\kappa, \tau)=$ $g_{a \gamma}^{2} h_{c}\left(k_{c}, \eta\right) / 2, \mathcal{R}(\kappa, \tau)=\left(g_{a \gamma}^{2} / k_{\max }\right) \rho_{c}\left(k_{c}, \eta\right)$, and $\Phi(\tau)=$ $2\left(k_{\max } g_{a \gamma} / \sigma_{c}\right) \varphi(\eta)$ with a change of the conformal time $\eta$ to the dimensionless one, $\tau=2 k_{\max }^{2} \eta / \sigma_{c}$, reads

$$
\begin{gathered}
\frac{\mathrm{d} \mathcal{H}}{\mathrm{d} \tau}=-\kappa^{2} \mathcal{H}(\kappa, \tau)+\frac{\mathrm{d} \Phi(\tau)}{\mathrm{d} \tau} \mathcal{R}(\kappa, \tau) \\
\frac{\mathrm{d} \mathcal{R}}{\mathrm{d} \tau}=-\kappa^{2} \mathcal{R}(\kappa, \tau)+\kappa^{2} \frac{\mathrm{d} \Phi(\tau)}{\mathrm{d} \tau} \mathcal{H}(\kappa, \tau) \\
\frac{\mathrm{d}^{2} \Phi}{\mathrm{d} \tau^{2}}+\frac{2}{a(\tau)} \frac{\mathrm{d} \Phi}{\mathrm{d} \tau}\left(\frac{\mathrm{d} a(\tau)}{\mathrm{d} \tau}+\frac{1}{2 a(\tau)} \int_{\kappa_{m}}^{1} \mathcal{R}(\kappa, \tau) d \kappa\right)+a^{2} \mu^{2} \Phi(\tau) \\
=\frac{1}{a^{2}(\tau)} \int_{\kappa_{m}}^{1} \kappa^{2} \mathcal{H}(\kappa, \tau) d \kappa
\end{gathered}
$$

In Eq. (2.9), $\mu=m_{a} \sigma_{c} / 2 k_{\max }^{2}$ is the dimensionless parameter given by the axion mass in Eq. (1.1). The lower limit $\kappa_{m}=k_{\min } / k_{\max }=k_{1} / k_{2}$ for the dimensionless Fourier momentum $\kappa=k_{c} / k_{\max }=k / k_{2}$ obeys the constraints

$$
2.6 \times 10^{-11} \leq \kappa_{m} \ll 1,
$$

where we account for Eq. (2.6).

Note that we have three free parameters for PMFs in our problem:

(1) A wide width of the Fourier spectrum for PMFs given by a small varying parameter $\kappa_{m}$ in Eq. (2.10), or by varying $k_{\max }=k_{\min } / \kappa_{m}$ for the fixed $k_{\min }=a\left(t_{0}\right) k_{1}$.

(2) The helicity parameter $q, 0 \leq|q| \leq 1$, entering the initial magnetic helicity spectrum [see Eq. (2.22) below], where $q=0$ corresponds to the nonhelical initial PMF, whereas $|q|=1$ corresponds to the maximum helical PMF.

(3) An undetermined cosmological magnetic field $B_{\text {now }}$ at the present redshift $z=0$ originating in our scenario from the initial PMF $B_{0}=B_{\text {now }}\left(1+z_{\mathrm{QCD}}\right)^{2}$ at $z_{\mathrm{QCD}}=4 \times 10^{11}$ in the early Universe.

These three parameters fully describe the necessary characteristics of PMFs: the spatial scale, its structure (the topology), and the strength.

Using the definition of the conformal time in Eq. (2.5), the scale in the radiation era, $a(t)=a\left(t_{0}\right) \sqrt{t / t_{0}}=$ $a\left(t_{0}\right)\left[1+a\left(t_{0}\right) \eta(t) / 2 t_{0}\right]$, can be rewritten in the new variables as $a(\tau)=a\left(t_{0}\right)[1+K \tau] \equiv a\left(t_{0}\right) \tilde{a}(\tau)$, with the factor

$$
\tilde{a}(\tau)=1+K \tau, \quad K=3.7 \times 10^{20} \kappa_{m}^{2},
$$

where $\tau \geq 0$ [13].

\section{A. Axion energy density}

The important characteristic of axions is the axion energy density $\rho_{a}(t)=T_{00}$, where $T_{00}$ is the component of the energy-momentum tensor for a scalar field,

$$
T_{\mu \nu}=\partial_{\mu} \varphi \partial_{\nu} \varphi-\frac{g_{\mu \nu}}{2}\left(g^{\lambda \rho} \partial_{\lambda} \varphi \partial_{\rho} \varphi-m_{a}^{2} \varphi^{2}\right) .
$$

In the case of a spatially homogeneous field, based on Eq. (2.12), one gets that

$\rho_{a}(t)=\frac{1}{2}\left[\dot{\varphi}^{2}+m_{a}^{2} \varphi^{2}\right]=\frac{1}{2}\left(\frac{k_{\max }}{g_{a \gamma} a}\right)^{2}\left[\left(\frac{\mathrm{d} \Phi}{\mathrm{d} \tau}\right)^{2}+\mu^{2} a^{2} \Phi^{2}(\tau)\right]$,

where $k_{\max }=k_{2} a=k_{1} a / \kappa_{m}$ is given by the parameter $\kappa_{m}$ in Eq. (2.10) and the minimal momentum $k_{1}$ in our causal scenario at $t_{0}=t_{\mathrm{QCD}}, k_{1}=l_{\mathrm{H}}\left(t_{0}\right)^{-1}=(2 / 3) \times 10^{-20} \mathrm{GeV}$. This results in a dimensional factor in front of the brackets in Eq. (2.13),

$$
C_{m}=\frac{1}{2}\left(\frac{k_{\max }}{g_{a \gamma} a}\right)^{2}=\frac{2}{9}\left(\frac{10^{-10}}{\kappa_{m}^{2}}\right) \mathrm{GeV}^{4},
$$

which depends on the PMF spectrum width $\kappa_{m}$.

\section{B. Initial conditions}

The initial axion zero mode amplitude $\varphi\left(t_{0}\right) \simeq f_{a}$, $\bar{\theta}\left(t_{0}\right) \simeq 1$ (see, e.g., Ref. [8]) corresponds to

$$
\Phi(\tau=0)=\frac{2 k_{\max } g_{a \gamma}}{\sigma_{c}} \varphi\left(t_{0}\right)=\frac{1.5 \times 10^{-24}}{\kappa_{m}},
$$

where $\kappa_{m}$ is the free parameter constrained in Eq. (2.10).

The initial derivative $\mathrm{d} \Phi /\left.\mathrm{d} \tau\right|_{\tau=0}$, which is needed to solve Eq. (2.9), is derived using the virial theorem $\left\langle\dot{\theta}^{2}\right\rangle=$ $m_{a}^{2}\left\langle\theta^{2}\right\rangle$ resulting from the axion energy density in Eq. (2.13) around its potential minimum [14],

$$
\rho=\frac{f_{a}^{2}}{2}\left[\dot{\theta}^{2}+m_{a}^{2}(t) \theta^{2}\right], \quad \theta(t)=\frac{\varphi}{f_{a}} .
$$

We approximate the initial value for the first derivative as $(\mathrm{d} \varphi / \mathrm{d} t)_{t=t_{0}}=m_{a} \varphi\left(t_{0}\right)$ relying on the virial theorem in Eq. (2.16). Then, one gets

$$
\left.\frac{\mathrm{d} \Phi}{\mathrm{d} \tau}\right|_{\tau=0}=\left(\frac{g_{a \gamma} a\left(t_{0}\right)}{k_{\max }}\right) m_{a} f_{a}=10^{3} \kappa_{m},
$$

where we substituted the "cold" axion mass $m_{a}$ in Eq. (1.1) at the moment of the QCD phase transition, $m_{a}(T) \rightarrow m_{a}$ at $T \rightarrow T_{\mathrm{QCD}}$. 


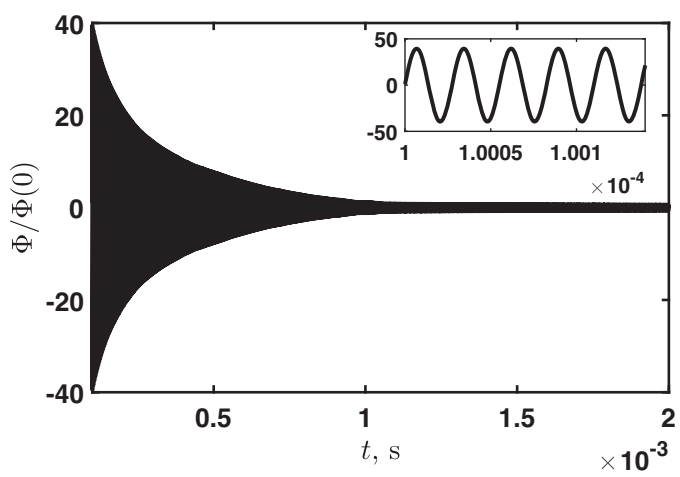

(a)

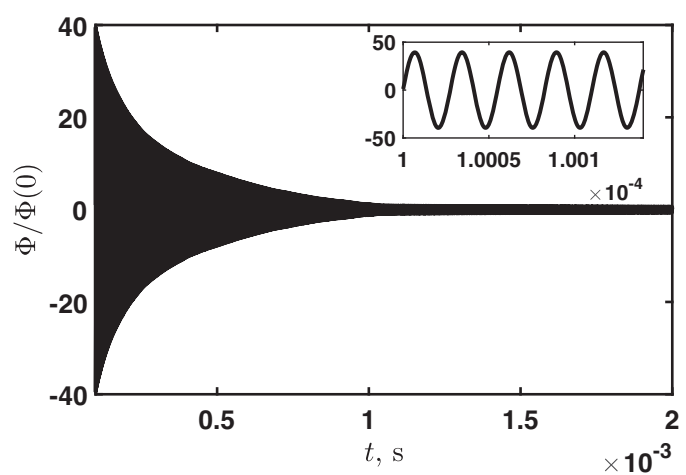

(b)

FIG. 1. (a) Evolution of the axion zero mode amplitude $\Phi(\tau) / \Phi(\tau=0) \equiv \varphi(t) / f_{a}=\bar{\theta}(t)$ for the Peccei-Quinn parameter $f_{\mathrm{PQ}} \simeq$ $f_{a}=10^{12} \mathrm{GeV}$ and Fourier spectrum width $\kappa_{m}=10^{-10}$ in Eq. (2.10), choosing the maximal $B_{\text {now }}=10^{-9} \mathrm{G}$. The seed magnetic field is supposed to be nonhelical, i.e., $q=0$ in Eq. (2.22). (b) Evolution of the axion zero mode amplitude $\Phi(\tau) / \Phi(\tau=0) \equiv \varphi(t) / f_{a}=\bar{\theta}(t)$ for the Peccei-Quinn parameter $f_{\mathrm{PQ}} \simeq f_{a}=10^{12} \mathrm{GeV}$ without the PMF contribution, $g_{a \gamma}=0$ and $q=0$.

Note that the mass term $(\mu a)^{2} \Phi$ in Eq. (2.9),

$$
(\mu a)^{2}=2.85 \times 10^{30} \kappa_{m}^{2}(1+K \tau)^{2},
$$

is rather large. We will see in Sec. III that this term is dominant.

The initial PMF spectra $\mathcal{R}(\kappa, \tau=0)$ and $\mathcal{H}(\kappa, \tau=0)$,

$$
\begin{gathered}
\mathcal{R}(\kappa, \tau=0)=\frac{g_{a \gamma}^{2}}{k_{\max }} \rho_{c}\left(k_{c}, \eta=0\right), \\
\mathcal{H}(\kappa, \tau=0)=\frac{g_{a \gamma}^{2}}{2} h_{c}\left(k_{c}, \eta=0\right),
\end{gathered}
$$

are given by the PMF spectra in the known form [15]

$$
\begin{gathered}
\rho_{c}\left(k_{c}, \eta=0\right)=C k_{c}^{n_{\mathrm{B}}}, \quad C=\frac{\left(n_{\mathrm{B}}+1\right) B_{\mathrm{now}}^{2}}{2 k_{\max }^{n_{\mathrm{B}}+1}\left(1-\kappa_{m}^{n_{\mathrm{B}}+1}\right)}, \\
h_{c}\left(k_{c}, \eta=0\right)=\frac{2 q}{k_{c}} \rho_{c}\left(k_{c}, \eta=0\right), \quad 0 \leq|q| \leq 1 .
\end{gathered}
$$

The constant $C$ for the spectrum in Eq. (2.21) is given by normalization on the initial magnetic energy density $B_{c}^{2}(\eta=0) / 2=\int \mathrm{d} k_{c} \rho_{c}\left(k_{c}, \eta=0\right)$ for the comoving PMF strength $B_{c}(\eta=0)=B_{\text {now }}$. Thus, by substituting Eqs. (2.21) and (2.22) into Eqs. (2.19) and (2.20), respectively, we obtain the initial PMF spectra

$$
\begin{gathered}
\mathcal{R}(\kappa, \tau=0)=\frac{C_{0}\left(n_{\mathrm{B}}+1\right)}{2\left(1-\kappa_{m}^{n_{\mathrm{B}}+1}\right)} \kappa^{n_{\mathrm{B}}}, \\
\mathcal{H}(\kappa, \tau=0)=q \frac{C_{0}\left(n_{\mathrm{B}}+1\right)}{2\left(1-\kappa_{m}^{n_{\mathrm{B}}+1}\right)} \kappa^{n_{\mathrm{B}}-1}, \quad C_{0}=\left(\frac{g_{a \gamma} B_{\text {now }}}{k_{\max }}\right)^{2} .
\end{gathered}
$$

We consider below the Kolmogorov spectrum with $n_{\mathrm{B}}=-5 / 3$.

\section{RESULTS}

In this section we present the numerical solution of Eqs. (2.7)-(2.9) with the initial conditions fixed in Sec. II B.

In Fig. 1(a) we rely on a maximum PMF value at present time of $B_{\text {now }}=10^{-9} \mathrm{G}$, which obeys the following bounds.

(1) $B_{1 \mathrm{Mpc}}<4.4 \times 10^{-9} \mathrm{G}$, which was found in Ref. [12] from CMB anisotropies (the effect on CMB polarization induced by Faraday rotation) for zero magnetic helicity $(q=0)$.

(2) $B_{1 \mathrm{Mpc}}<5.6 \times 10^{-9}$ G for a maximally helical magnetic field $(q=1)$.

Notice that, at the initial time $t_{0}=t_{\mathrm{QCD}}$ chosen in our problem for the causal scenario, the maximum spatial PMF scale $\Lambda_{\mathrm{B}}^{(\max )}=l_{\mathrm{H}}\left(t_{0}\right) \simeq 3 \times 10^{6} \mathrm{~cm}$. This spatial scale occurs rather small after the Universe expands until the present time. It grows up to the scale $\Lambda_{\mathrm{B}}(z=0) \simeq 1 \mathrm{pc}$ only because the horizon expands during the cooling much faster $\left(l_{\mathrm{H}} \sim T^{-2}\right)$ than the correlation length $\left(\Lambda_{\mathrm{B}} \sim T^{-1}\right)$. However, the inverse cascade in relativistic magnetohydrodynamics (MHD), accounting for nonlinear terms in the Navier-Stokes equation (we do not address the full MHD approach here), can rearrange the Fourier MHD spectra in such a way that $\Lambda_{\mathrm{B}}$ as a measure of the coherence length of the magnetic field can increase in coherence by 5 orders of magnitude [16]. This fact allows us to get closer to the present scale $\Lambda_{\mathrm{B}} \sim 1 \mathrm{Mpc}$ for PMFs bounded in Ref. [12]. The other problem is that, around the time of recombination, the photon diffusion becomes very large and the socalled Silk mechanism could destroy the PMF characteristics. This danger was refuted in Ref. [17], where nonlinear effects were shown to most likely prevent this problem from happening. 


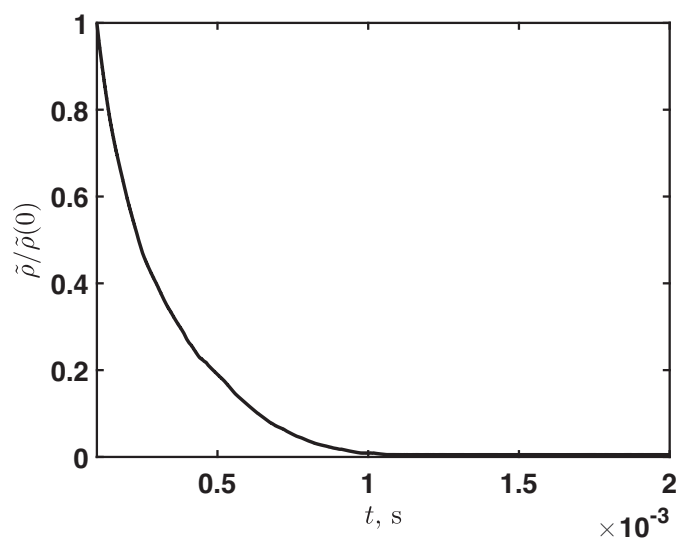

(a)

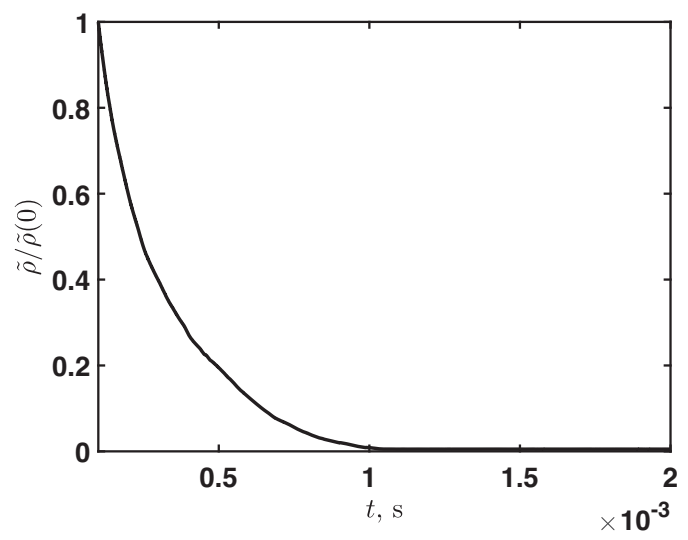

(b)

FIG. 2. (a) Evolution of the axion energy density $\tilde{\rho}(\tau) / \tilde{\rho}(\tau=0)$ for the Peccei-Quinn parameter $f_{\mathrm{PQ}} \simeq f_{a}=10^{12} \mathrm{GeV}$ and Fourier spectrum width $\kappa_{m}=10^{-10}$ in Eq. (2.10), choosing the maximal $B_{\text {now }}=10^{-9} \mathrm{G}$. The seed magnetic field is supposed to be nonhelical, i.e., $q=0$ in Eq. (2.22). (b) Evolution of the axion energy amplitude $\tilde{\rho}(\tau) / \tilde{\rho}(\tau=0)$ for the Peccei-Quinn parameter $f_{\mathrm{PQ}} \simeq f_{a}=$ $10^{12} \mathrm{GeV}$ without the PMF contribution, $g_{a \gamma}=0$ and $q=0$.

In Fig. 1(a) the normalized axion field $\Phi(\tau) / \Phi(0)=$ $\varphi(t) / \varphi\left(t_{0}\right)=\bar{\theta}(t)$ grows 40 times starting from $\bar{\theta}\left(t_{0}\right)=$ $\varphi\left(t_{0}\right) / f_{a}=1$ during a short time [see the inset in Fig. 1(a)]. Then it reduces quickly to the same value $\bar{\theta}(t) \sim 1$ acceptable for a future axion contribution to CDM. This result does not depend on the presence of PMFs; see Fig. 1 (b) where we put $g_{a \gamma}=0$ and $q=0$ to exclude interactions of axions with the PMFs. In both cases, this evolution of $\bar{\theta}(t)$ amplitudes happens during the lepton era in the hot Universe plasma.

One can explain the appearance of a large amplitude $\Phi(\tau) / \Phi(0) \sim 40$ just after the start at $t \geq t_{0}=10^{-4} \mathrm{~s}$ using the initial conditions in Eqs. (2.15)-(2.18). For a slow change of the scale factor $a(\tau)$ and neglecting the PMF terms, we get oscillations of the axion field $\Phi(\tau)=$ $\mathcal{A} \cos \omega \tau+\mathcal{B} \sin \omega \tau$ that obey the simplified equation in Eq. (2.9), $\Phi_{\tau \tau}+\omega^{2} \Phi(\tau)=0, \omega=\mu a, \tau \ll 1$. Since $\mathcal{A}=$ $\Phi(0)$ and $\mathcal{B}=\mathrm{d} \Phi /\left.\mathrm{d} \tau\right|_{\tau=0} / \omega$, one finds from the initial conditions the corresponding ratio $\Phi(\tau) / \Phi(0)$ for the amplitude of $\Phi(\tau)$ at $\omega \tau=\pi / 2$,

$$
\begin{gathered}
\frac{\Phi(\tau)}{\Phi(0)}=\frac{\mathcal{B}}{\mathcal{A}}, \quad \text { or } \\
\frac{\Phi(\tau)}{\Phi(0)}=\frac{(\mathrm{d} \Phi / \mathrm{d} \tau)_{\tau=0}}{\Phi(0)(\mu a)}=\frac{10^{3} \kappa_{m}}{1.5 \times 10^{-24}\left(\sqrt{2.85} \times 10^{15}\right)} \simeq 40,
\end{gathered}
$$

where we substituted the values of the parameters from Eqs. (2.15)-(2.18) and put $\kappa_{m}=10^{-10}$.

In what follows, the phase $\bar{\theta}=\varphi(t) / f_{a}=\Phi(\tau) / \Phi(0)$ oscillates and diminishes with the Universe's expansion as $\sim t^{-3 / 4}$ [see Eq. (45) in Ref. [10]] for a zero mode evolution neglecting electromagnetism for axions, or as $\sim 1 / a(\eta) \sqrt{a(\eta)}$ during the radiation era, $a \sim \sqrt{t}$ (see p. 40 of Ref. [7]). Of course, the oscillation frequency $\omega(\tau)=$ $\mu a(\tau)$ rises with the expansion. It makes rapid oscillations in Fig. 1 indistinguishable for times $t \gg t_{0}$. Distinguishable oscillations with the large amplitude $\Phi / \Phi(0) \sim 40$ at $t \sim t_{0}$ are shown in the insets in Fig. 1.

The more smooth evolution curves are seen in Fig. 2 for the normalized axion energy density $\rho_{a}(t)=C_{m} \tilde{\rho}(\tau)$ given by Eqs. (2.13) and (2.14). The ratio $\rho_{a}(t) / \rho\left(t_{0}\right)$ corresponds to the ratio of the dimensionless functions $\tilde{\rho}(\tau)$,

$$
\tilde{\rho}(\tau)=\left[\left(\frac{\mathrm{d} \Phi}{\mathrm{d} \tau}\right)^{2}+\mu^{2} a^{2} \Phi^{2}(\tau)\right]
$$

and $\tilde{\rho}(0)$ plotted in Fig. 2,

$$
\frac{\tilde{\rho}(\tau)}{\tilde{\rho}(0)}=\frac{(\mathrm{d} \Phi / \mathrm{d} \tau)^{2}+\mu^{2} a^{2} \Phi^{2}(\tau)}{(\mathrm{d} \Phi / \mathrm{d} \tau)_{\tau=0}^{2}+\mu^{2} a^{2}(0) \Phi^{2}(0)} .
$$

For $\kappa_{m}=10^{-10}$ the huge dimensional factor $C_{m}=(2 / 9) \times$ $10^{10} \mathrm{GeV}^{4}$ in Eq. (2.14) is compensated in the product $\rho_{a}\left(t_{0}\right)=C_{m} \tilde{\rho}(\tau=0)$ by the small value $\tilde{\rho}(\tau=0)=10^{-14}$ calculated numerically from the system of evolution equations (2.7)-(2.9), or the initial axion energy density $\rho_{a}\left(t_{0}\right)=C_{m} \tilde{\rho}(\tau=0)=(2 / 9) \times 10^{-4} \mathrm{GeV}^{4}$. On the other hand, the ultrarelativistic matter energy density $\rho=$ $\pi^{2} T^{4} g^{*} / 90$ in Friedmann's law is given by the number of relativistic degrees of freedom $g^{*}=10.75$ and temperature $T_{\mathrm{QCD}}=0.1 \mathrm{GeV}$ at the same moment $t_{0}, \rho=$ $1.18 \times 10^{-4} \mathrm{GeV}^{4}$. Thus, at the start we get a remarkable ratio of energy densities for the axion as a candidate for $\mathrm{CDM}, \rho_{a}\left(t_{0}\right) / \rho=0.19$. Notice that this ratio does not depend on a width of the PMF spectrum $\kappa_{m}$ in Eq. (2.10). 
Then, the axion energy density $\rho_{a} \sim \tilde{\rho}(\tau)$ decreases as seen in Fig. 2, and the matter density $\rho$ decreases due to the Universe cooling, $\rho \sim T^{4}$, therefore supporting approximately the same ratio $\rho_{a}(t) / \rho(t) \simeq 0.2$.

We are not guaranteed to observe the maximum PMF $B_{\text {now }}=10^{-9} \mathrm{G}$ in the future, while some predictions with a lower bound for $B_{1 \mathrm{Mpc}}(z=0)>10^{-16} \mathrm{G}$ exist [18]. They arise, in particular, from $\gamma$-ray observations in the Fermi experiment [19].

Recently, in Ref. [20] it was suggested to look for PMFs at a level of the comoving strength $B_{\text {now }}=B a^{2}=$ const $\simeq$ $10^{-11} \mathrm{G}$. In the last photon scattering (for which $\mathrm{CMB}$ instruments are sensitive), such a field corresponds to the PMF strength $B\left(z_{r}\right)=B_{\text {now }}\left(1+z_{r}\right)^{2} \simeq 10^{-5} \mathrm{G}$, whereas, in our problem, it corresponds to $B_{0}=B_{\text {now }}\left(1+z_{\mathrm{QCD}}\right)^{2} \simeq$ $1.6 \times 10^{12} \mathrm{G}$.

\section{DISCUSSION}

In the present work we have studied the evolution of a zero axion mode, $\nabla \varphi=0$, in a hot plasma of the early Universe in the presence of PMFs that do not exceed $B_{\text {now }}=10^{-9} \mathrm{G}$ at the present time. We concluded that such a mode can contribute to the axion CDM since its amplitude tends to $\bar{\theta}(t)=\varphi(t) / f_{a} \rightarrow 1$ when evolving during the radiation era; see Fig. 1. Simultaneously, the corresponding axion energy density $\rho_{a}(t)$ decreases in such a way that its part in the total matter energy density $\rho$ tends to the CDM ratio $\rho_{a}(t) / \rho \simeq 0.2$; see comments after Eq. (3.3) concerning the axion energy evolution in Fig. 2.

Notice that our PMF amplitudes $\sqrt{\int_{\kappa_{m}}^{1} \mathrm{~d} \kappa \mathcal{R}(\kappa, \tau)} \sim$ $B a^{2}=$ const correspond to the comoving strength for a given $B_{\text {now }}=B a^{2}=$ const and $\kappa_{m}$. Therefore, the ratio

$$
\sqrt{\frac{\int_{\kappa_{m}}^{1} \mathrm{~d} \kappa \mathcal{R}(\kappa, \tau)}{\int_{\kappa_{m}}^{1} \mathrm{~d} \kappa \mathcal{R}(\kappa, \tau=0)}} \approx 1
$$

does not depend on the conformal time $\eta \sim \tau$, being slightly violated by PMF interactions with axions. A similar conservation concerns the magnetic helicity density, $\int_{\kappa_{m}}^{1} \tilde{\mathcal{H}}(\kappa, \tau) \mathrm{d} \kappa \sim h a^{3}=$ const, calculated from the same self-consistent system of equations (2.7)-(2.9) for each given $B_{\text {now }}$ and $\kappa_{m}$. Hence, the magnetic helicity density for the case $q=0$,

$$
\int_{\kappa_{m}}^{1} \mathcal{H}(\kappa, \tau) \mathrm{d} \kappa \approx \int_{\kappa_{m}}^{1} \mathcal{H}(\kappa, \tau=0) \mathrm{d} \kappa=0,
$$

does not depend on time as well. A similar time independence occurs in the case of a maximally helical PMF with $q=1$. For the PMF amplitude one obtains precisely the result in Eq. (4.1), while the magnetic helicity density in Eq. (4.2) changes to

$$
\int_{\kappa_{m}}^{1} \mathcal{H}(\kappa, \tau) \mathrm{d} \kappa \approx \int_{\kappa_{m}}^{1} \mathcal{H}(\kappa, \tau=0) \mathrm{d} \kappa=\text { const }
$$

where the constant depends on the initial conditions.

The conservation of the magnetic helicity density in Eq. (4.3) is not surprising in the absence of axion interactions with PMFs through the Primakoff effect. Indeed, the comoving mean energy density $\varepsilon_{\mathrm{M}}=\left\langle\mathbf{B}^{2}\right\rangle / 2=$ $\int \mathrm{d} k_{c} \rho_{c}\left(k_{c}, \eta\right)$ decays with time such that $\varepsilon_{\mathrm{M}} \propto \eta^{-2 / 3}$, while the correlation length grows like $\xi_{\mathrm{M}} \propto \eta^{2 / 3}$, as it follows from the dimensionless analysis based on the Kolmogorovtype approach for the magnetic turbulence; see, e.g., Refs. [21,22]. This results in the conservation of the magnetic helicity density, $h_{c}(\eta)=\int \mathrm{d} k_{c} h_{c}\left(k_{c}, \eta\right)=\left\langle\mathbf{B}^{2}\right\rangle \xi_{\mathrm{M}}=$ const. If we additionally switch on the interaction of axions with PMFs, the violation of that conservation law is negligible because of a very small coupling constant $g_{a \gamma}=\alpha_{\mathrm{em}} / 2 \pi f_{a}$; cf. Eqs (4.2) and (4.3).

Equations (4.1)-(4.3) were directly checked by our numerical simulations. Thus, we confirm the conclusion of Ref. [8] that the axion zero mode $(\nabla \varphi=0)$ practically does not influence any PMF characteristics. The main discrepancies between our work and Ref. [8] consist in the exact accounting for the Universe's expansion in the dynamics of axions and electromagnetic fields. We have also taken into account the nontrivial spectra of the magnetic helicity and magnetic energy densities.

At first glance, there is a similarity between the axion electromagnetism and the chiral magnetic effect (CME) $[23,24]$, which was a motivation for us to apply the Primakoff mechanism and study the mutual influence of an axion zero mode and PMFs in the early Universe.

However, it is not surprising that the CME is significantly more efficient for the amplification of PMFs than in the case of the axion interactions with magnetic fields. In the induction equation (1.2), the helicity parameter $\alpha(t)=\left(g_{a \gamma} \mathrm{d} \varphi / \mathrm{d} t\right) / \sigma_{\text {cond }}$ that enters the PMF instability term $\alpha(t)(\nabla \times \mathbf{B})$ is much less than the corresponding CME parameter $\alpha_{\mathrm{CME}}(t)=2 \alpha_{\mathrm{em}} \mu_{5}(t) / \pi \sigma_{\text {cond }}$, where $\mu_{5}=$ $\left(\mu_{\mathrm{R}}-\mu_{\mathrm{L}}\right) / 2$ and $\mu_{\mathrm{R}, \mathrm{L}}$ are the chemical potentials for right and left charged leptons. Indeed, the CME parameter $\alpha_{\mathrm{CME}}$ is given by a large pseudoscalar $\mu_{5} / T \sim 10^{-5}$ at the temperature $T_{\mathrm{QCD}} \sim 10^{8} \mathrm{eV}$, or $\mu_{5} \sim 10^{3} \mathrm{eV}$ (see Fig. 1 in Ref. [23]). However, in the case of axions the pseudoscalar $\alpha$, when accounting for the initial derivative $\mathrm{d} \varphi /\left.\mathrm{d} t\right|_{t=t_{0}} \simeq m_{a} \varphi\left(t_{0}\right)=m_{a} f_{a}$ [see below Eq. (2.15)], is estimated as

$$
\alpha=\left(\frac{\alpha_{\mathrm{em}}}{2 \pi}\right) \frac{m_{a}}{\sigma_{\mathrm{cond}}} \ll \alpha_{\mathrm{CME}}=\left(\frac{2 \alpha_{\mathrm{em}}}{\pi}\right) \frac{\mu_{5}}{\sigma_{\mathrm{cond}}},
$$

where, obviously, the axion mass $m_{a}$ in Eq. (1.1) is quite small, $m_{a} \ll \mu_{5}$. 
Thus, we conclude that the axion zero mode $\nabla \varphi=0$ and PMFs evolve independently of each other. Second, for reasonable PMF characteristics, the axion zero mode that appears in the case when the inflation occurs after the Peccei-Quinn phase transition, $T_{\mathrm{PQ}}>T_{\mathrm{R}}, T_{\mathrm{PQ}} \simeq 10^{12} \mathrm{GeV}$, can be a candidate for CDM. If there is no inflation after the PQ phase transition, $T_{\mathrm{PQ}}<T_{\mathrm{R}}$, the axion field is spatially varying, $\nabla \varphi \neq 0$. Axion strings can produce such nonzero momentum modes of the axion field which could contribute to the CDM [10,25]. The interaction of these modes with PMFs is beyond scope of the present work.

\section{ACKNOWLEDGMENTS}

We are grateful to V. A. Berezin for remarks concerning the description of massive scalar fields in general relativity with conformal variables.

\section{APPENDIX: COMPLETE SET OF EVOLUTION EQUATIONS FOR THE AXION ZERO MODE AND PMF SPECTRA}

In this Appendix, we derive the equations for the spectra of the magnetic energy and helicity, as well as for the axion field.

The action for the axion field $\varphi$ with mass $m_{a}$ interacting with the electromagnetic field $A^{\mu}$ in curved spacetime is

$$
\begin{aligned}
S= & \int \mathrm{d}^{4} x \sqrt{-g}\left[\frac{1}{2}\left(g^{\mu \nu} \partial_{\mu} \varphi \partial_{\nu} \varphi-m_{a}^{2} \varphi^{2}\right)-\frac{1}{4} F_{\mu \nu} F_{\lambda \rho} g^{\mu \lambda} g^{\nu \rho}\right. \\
& \left.-\frac{g_{a \gamma} \varphi}{4} F_{\mu \nu} \tilde{F}_{\lambda \rho} g^{\mu \lambda} g^{\nu \rho}-A_{\mu} J_{\nu} g^{\mu \nu}\right],
\end{aligned}
$$

where $g=\operatorname{det}\left(g_{\mu \nu}\right), g_{\mu \nu}$ is the metric tensor, $F_{\mu \nu}=\partial_{\mu} A_{\nu}-$ $\partial_{\nu} A_{\mu}$ is the electromagnetic stress tensor, $g_{a \gamma}$ is the coupling constant, $\tilde{F}^{\mu \nu}=\frac{1}{2} E^{\mu \nu \alpha \beta} F_{\alpha \beta}$ is the dual counterpart of $F_{\mu \nu}$, $E^{\mu \nu \alpha \beta}=\frac{1}{\sqrt{-g}} \varepsilon^{\mu \nu \alpha \beta}$ is the covariant antisymmetric tensor, $\varepsilon^{0123}=+1$, and $J^{\mu}$ is the external current.

Using Eq. (A1), one gets the equations for $\varphi$ and $A^{\mu}$ in the form

$$
\begin{array}{r}
\frac{1}{\sqrt{-g}} \partial_{\mu}\left(\sqrt{-g} \partial^{\mu} \varphi\right)+m_{a}^{2} \varphi+\frac{g_{a \gamma}}{4} F_{\mu \nu} \tilde{F}^{\mu \nu}=0, \\
\frac{1}{\sqrt{-g}} \partial_{\nu}\left(\sqrt{-g} F^{\mu \nu}\right)+\partial_{\nu} \varphi \tilde{F}^{\mu \nu}+J^{\mu}=0 .
\end{array}
$$

In Eq. (A3) we take into account that $\nabla_{\nu} \tilde{F}^{\mu \nu}=0$, where $\nabla_{\mu}$ is the covariant derivative. This fact can be proven based on the Maxwell equation, $\nabla_{\mu} F_{\alpha \beta}+\nabla_{\alpha} F_{\beta \mu}+\nabla_{\beta} F_{\mu \alpha}=0$, and the definition of $\tilde{F}^{\mu \nu}$.

Now we choose the FRW metric, which has the form

$$
g_{\mu \nu}=\operatorname{diag}\left(1,-a^{2},-a^{2},-a^{2}\right),
$$

where $a=a(t)$ is the scale factor. In this case, $\sqrt{-g}=a^{3}$. We also take the zero-mode approximation for the axion field, $\partial_{0} \varphi \neq 0$ and $\partial_{i} \varphi=0$. The four-current $J^{\mu}$ in Eq. (A3) can be represented in the form $J^{\mu}=(\rho, \mathbf{J} / a)$, where $\rho$ is the charge density and $\mathbf{J}$ is the electric three-current.

Following Ref. [16], we introduce the conformal variables

$\mathbf{E}_{c}=a^{2} \mathbf{E}, \quad \mathbf{B}_{c}=a^{2} \mathbf{B}, \quad \rho_{c}=a^{3} \rho, \quad \mathbf{J}_{c}=a^{3} \mathbf{J}$.

Then, Eq. (A3) takes the form

$$
\begin{aligned}
& \left(\nabla \cdot \mathbf{E}_{c}\right)=\rho_{c}, \quad \frac{\partial \mathbf{E}_{c}}{\partial \eta}+\mathbf{J}_{c}+g_{a \gamma} \mathbf{B}_{c} \frac{\mathrm{d} \varphi}{\mathrm{d} \eta}=\left(\nabla \times \mathbf{B}_{c}\right), \\
& \left(\nabla \cdot \mathbf{B}_{c}\right)=0, \quad \frac{\partial \mathbf{B}_{c}}{\partial \eta}=-\left(\nabla \times \mathbf{E}_{c}\right),
\end{aligned}
$$

where $\eta=\int \frac{\mathrm{d} t}{a}$ is the conformal time. Equation (A6) should be supplied with the relation between $\mathbf{E}_{c}$ and $\mathbf{J}_{c}: \mathbf{J}_{c}=\sigma_{c} \mathbf{E}_{c}$, where $\sigma_{c}=a \sigma=\mathrm{const}$ and $\sigma$ is the conductivity.

After the volume integration, $V^{-1} \int \mathrm{d}^{3} x(\ldots)$, Eq. (A2) for the axion zero mode can be rewritten in the form

$\frac{\mathrm{d}^{2} \varphi}{\mathrm{d} \eta^{2}}+\frac{2}{a} \frac{\mathrm{d} a}{\mathrm{~d} \eta} \frac{\mathrm{d} \varphi}{\mathrm{d} \eta}+m_{a}^{2} a^{2} \varphi=\frac{g_{a \gamma}}{V a^{2}} \int \mathrm{d}^{3} x\left(\mathbf{E}_{c}(\eta, \mathbf{x}) \cdot \mathbf{B}_{c}(\eta, \mathbf{x})\right)$,

where we substituted $F_{\mu \nu} \tilde{F}^{\mu \nu}=-4(\mathbf{E} \mathbf{B})$ by multiplying Eq. (A2) by $a^{2}$, and assumed that $\varphi$ does not change under the conformal transformations. The right-hand side of Eq. (A7) enters our master Eq. (2.1) as

$$
-\frac{g_{a \gamma}}{2 a^{2}} \frac{\mathrm{d} h_{c}(\eta)}{\mathrm{d} \eta}=\frac{g_{a \gamma}}{V a^{2}} \int \mathrm{d}^{3} x\left(\mathbf{E}_{c}(\eta, \mathbf{x}) \cdot \mathbf{B}_{c}(\eta, \mathbf{x})\right) .
$$

Such a change follows from the definition of the conformal PMF helicity density $h_{c}=a^{3} h=V^{-1} \int \mathrm{d}^{3} x \mathbf{A}_{c}(\eta, \mathbf{x})$. $\mathbf{B}_{c}(\eta, \mathbf{x})$ obeying the Maxwell equation (A6). Here $h_{c}(\eta)=$ $\int \mathrm{d} k_{c} h_{c}\left(k_{c}, \eta\right)$ is given by the spectrum of the PMF helicity.

We introduce the conformal spectra of the PMF energy and PMF helicity density,

$$
\begin{aligned}
& \rho_{c}\left(k_{c}, \eta\right)=\frac{k_{c}^{2}}{4 \pi^{2} V} \mathbf{B}_{c}\left(k_{c}, \eta\right) \cdot \mathbf{B}_{c}^{*}\left(k_{c}, \eta\right), \\
& h_{c}\left(k_{c}, \eta\right)=\frac{k_{c}^{2}}{2 \pi^{2} V} \mathbf{A}_{c}\left(k_{c}, \eta\right) \cdot \mathbf{B}_{c}^{*}\left(k_{c}, \eta\right),
\end{aligned}
$$

where $k_{c}=k a$ is the conformal momentum, $\mathbf{A}_{c}\left(k_{c}, \eta\right)$ and $\mathbf{B}_{c}\left(k_{c}, \eta\right)$ are the Fourier components of the vector potential and magnetic field, and $V$ is the normalization volume.

Based on Eqs. (A6) and (A7), as well as using the results of Ref. [26], we get that 


$$
\begin{aligned}
& \frac{\partial h_{c}\left(k_{c}, \eta\right)}{\partial \eta}=-\frac{2 k_{c}^{2}}{\sigma_{c}} h_{c}\left(k_{c}, \eta\right)+\frac{4 g_{a \gamma}}{\sigma_{c}} \frac{\mathrm{d} \varphi(\eta)}{\mathrm{d} \eta} \rho_{c}\left(k_{c}, \eta\right), \\
& \frac{\partial \rho_{c}\left(k_{c}, \eta\right)}{\partial \eta}=-\frac{2 k_{c}^{2}}{\sigma_{c}} \rho_{c}\left(k_{c}, \eta\right)+k_{c}^{2} \frac{g_{a \gamma}}{\sigma_{c}} \frac{\mathrm{d} \varphi(\eta)}{\mathrm{d} \eta} h_{c}\left(k_{c}, \eta\right),
\end{aligned}
$$

and

$$
\begin{aligned}
& \frac{\mathrm{d}^{2} \varphi(\eta)}{\mathrm{d} \eta^{2}}+\frac{2}{a(\eta)} \frac{\mathrm{d} \varphi(\eta)}{\mathrm{d} \eta}\left(\frac{\mathrm{d} a(\eta)}{\mathrm{d} \eta}+\frac{g_{a \gamma}^{2}}{a(\eta) \sigma_{c}} \int_{k_{\min }}^{k_{\max }} \rho_{c}\left(k_{c}, \eta\right) \mathrm{d} k_{c}\right) \\
& +m_{a}^{2} a^{2}(\eta) \varphi(\eta)=\frac{g_{a \gamma}}{a^{2}(\eta) \sigma_{c}} \int_{k_{\text {min }}}^{k_{\max }} k_{c}^{2} h_{c}\left(k_{c}, \eta\right) \mathrm{d} k_{c},
\end{aligned}
$$

where $k_{\min \text { max }}$ are the ranges of the $k_{c}$ variation.

Now we define the dimensionless variables

$$
\kappa=\frac{k_{c}}{k_{\max }}, \quad \tau=\frac{2 k_{\max }^{2}}{\sigma_{c}} \eta,
$$

and the dimensionless functions of these variables

$$
\begin{aligned}
\mathcal{H}(\kappa, \tau) & =\left(\frac{g_{a \gamma}^{2}}{2}\right) h_{c}\left(k_{c}, \eta\right), \quad \mathcal{R}(\kappa, \tau)=\left(\frac{g_{a \gamma}^{2}}{k_{\max }}\right) \rho_{c}\left(k_{c}, \eta\right), \\
\Phi(\tau) & =\left(\frac{2 k_{\max } g_{a \gamma}}{\sigma_{c}}\right) \varphi(\eta) .
\end{aligned}
$$

Equations (A10) and (A11) can be rewritten in the form

$$
\begin{aligned}
& \frac{\partial \mathcal{H}(\tau, \kappa)}{\partial \tau}=-\kappa^{2} \mathcal{H}(\tau, \kappa)+\frac{\mathrm{d} \Phi(\tau)}{\mathrm{d} \tau} \mathcal{R}(\tau, \kappa), \\
& \frac{\partial \mathcal{R}(\tau, \kappa)}{\partial \tau}=-\kappa^{2} \mathcal{R}(\tau, \kappa)+\kappa^{2} \frac{\mathrm{d} \Phi(\tau)}{\mathrm{d} \tau} \mathcal{H}(\tau, \kappa), \\
& \frac{\mathrm{d}^{2} \Phi(\tau)}{\mathrm{d} \tau^{2}}+\frac{2}{a(\tau)} \frac{\mathrm{d} \Phi(\tau)}{\mathrm{d} \tau}\left(\frac{\mathrm{d} a(\tau)}{\mathrm{d} \tau}+\frac{1}{2 a(\tau)} \int_{\kappa_{m}}^{1} \mathcal{R}(\tau, \kappa) \mathrm{d} \kappa\right) \\
&=-\mu^{2} a^{2}(\tau) \Phi(\tau)+\frac{1}{a^{2}(\tau)} \int_{\kappa_{m}}^{1} \kappa^{2} \mathcal{H}(\tau, \kappa) \mathrm{d} \kappa,
\end{aligned}
$$

where $\mu=m_{a} \sigma_{c} / 2 k_{\max }^{2}$ and $\kappa_{m}=k_{\min } / k_{\max }$.
[1] R. D. Peccei and H. R. Quinn, $C P$ Conservation in the Presence of Pseudoparticles, Phys. Rev. Lett. 38, 1440 (1977).

[2] S. Weinberg, A New Light Boson?, Phys. Rev. Lett. 40, 223 (1978).

[3] F. Wilczek, Problem of Strong $P$ and $T$ Invariance in the Presence of Instantons, Phys. Rev. Lett. 40, 279 (1978).

[4] A. Ringwald, Exploring the role of axions and other WISPs in the dark universe, Phys. Dark Universe 1, 116 (2012).

[5] P. W. Graham, I. G. Irastorza, S. K. Lamoreaux, A. Lindner, and K. A. van Bibber, Experimental searches for the axion and axion-like particles, Annu. Rev. Nucl. Part. Sci. 65, 485 (2015).

[6] E. Aprile et al. (XENON Collaboration), Excess electronic recoil events in XENON1T, Phys. Rev. D 102, 072004 (2020).

[7] D. S. Gorbunov and V. A. Rubakov, Introduction to the Theory of the Early Universe: Hot Big Bang Theory (World Scientific, Singapore, 2011).

[8] A. Long and T. Vachaspati, Implications of a primordial magnetic field for magnetic monopoles, axions, and dirac neutrinos, Phys. Rev. D 91, 103522 (2015).

[9] If the inflation occurs with the reheating temperature, $T_{\mathrm{R}}$, smaller than $T_{\mathrm{PQ}} \sim f_{a}, T_{\mathrm{R}}<T_{\mathrm{PQ}}$, the axion field is homogenized over enormous distances. The subsequent evolution of this zero momentum mode is relatively simple in comparison with the multimode case, in which $\nabla \varphi \neq 0$ at $T_{\mathrm{R}}>T_{\mathrm{PQ}}$. In this case, strings, domains as topological defects in the axion field are present [10].
[10] P. Sikivie, Axion cosmology, Lect. Notes Phys. 741, 19 (2008).

[11] Accounting for the scale factor $a=(1+z)^{-1}$, we vary the undetermined PMF strength $B_{c}=a^{2} B=B_{\text {now }}$, where $B_{\text {now }}(z=0) \leq 10^{-9} \mathrm{G}$ for extragalactic fields [12]. For the initial time at the QCD phase transition the PMF occurs much stronger, $B_{c}\left(t_{0}\right)=B_{\text {now }}\left(1+z_{\mathrm{QCD}}\right)^{2}$.

[12] P. A. R. Ade et al. (Plank Collaboration), Plank 2015 results. XIX. Constraints on primordial magnetic field, Astron. Astrophys. 594, A19 (2016).

[13] For example, substituting the parameter $\kappa_{m}=10^{-10}$ obeying inequalities in Eq. (2.10) one gets $\tilde{a}=1+3.7 \tau$.

[14] L. Duffy and K. Bibber, Axions as dark matter particles, New J. Phys. 11, 105008 (2009).

[15] The equivalence to physical Fourier variables for the corresponding PMF spectrum $\rho_{\mathrm{B}}\left(k, t_{0}\right)=C_{1} k^{n_{\mathrm{B}}}$ comes from its normalization on $B^{2}\left(t_{0}\right) / 2=B_{\text {now }}^{2} / 2 a^{4}\left(t_{0}\right)$ and the relation $\rho_{c}(\eta=0)=a^{4}\left(t_{0}\right) \rho_{\mathrm{B}}\left(t_{0}\right)$ for the conformal PMF energy density $\rho_{c}(\eta=0)$ and the standard one, $\rho_{\mathrm{B}}\left(t_{0}\right)=$ $\int_{k_{1}}^{k_{2}} \mathrm{~d} k \rho_{\mathrm{B}}\left(k, t_{0}\right)$, where $k$ are physical momenta, $k=k_{c} / a$.

[16] A. Brandenburg, K. Enqvist, and P. Olesen, Large-scale magnetic fields from hydromagnetic turbulence in the very early universe, Phys. Rev. D 54, 1291 (1996).

[17] A. Brandenburg, K. Enqvist, and P. Olesen, The Effect of Silk damping on primordial magnetic fields, Phys. Lett. B 392, 395 (1997).

[18] A. Neronov and I. Vovk, Evidence for strong extragalactic magnetic fields from Fermi observations of $\mathrm{TeV}$ blazars, Science 328, 73 (2010). 
[19] W. B. Atwood et al., The large area telescope on the Fermi gamma-ray space telescope mission, Astrophys. J. 697, 1071 (2009).

[20] K. Jedamzik and L. Pogosian, Reliving the Hubble Tension with Primordial Magnetic Fields, Phys. Rev. Lett. 125, 181302 (2020).

[21] T. Kahniashvili, A. G. Tevzadze, A. Brandenburg, and A. Neronov, Evolution of primordial magnetic fields from phase transitions, Phys. Rev. D 87, 083007 (2013).

[22] A. Brandenburg, T. Kahniashvili, S. Mandal, A. R. Pol, A. G. Tevladze, and T. Vachaspati, Evolution of hydromagnetic turbulence from the electroweak phase transition, Phys. Rev. D 96, 123528 (2017).
[23] A. Boyarsky, J. Fröhlich, and O. Ruchayskiy, SelfConsistent Evolution of Magnetic Fields and Chiral Asymmetry in the Early Universe, Phys. Rev. Lett. 108, 031301 (2012).

[24] H. Tashiro, T. Vachaspati, and A. Vilenkin, Chiral effects and cosmic magnetic fields, Phys. Rev. D 86, 105033 (2012).

[25] S. Davidson, Axions: Bose Einstein condensate or classical field?, Astropart. Phys. 65, 101 (2015).

[26] M. Dvornikov and V. B. Semikoz, Instability of magnetic fields in electroweak plasma driven by neutrino asymmetries, J. Cosmol. Astropart. Phys. 05 (2014) 002. 